\title{
Central Vision Loss Associated with Silicone Oil in the Treatment of Retinal Detachment
}

\author{
Nicholas B Grissom ${ }^{1 *}$, Vishak J. John*, Timothy Martin ${ }^{1}$ \\ ${ }^{1}$ Wake Forest School of Medicine, Medical Center Boulevard Winston-Salem, N.C
}

\author{
Received: 12 August, 2016; Accepted: 19 September, 2016; Published: 29 September, 2016 \\ *Corresponding author: Vishak J. John, MD, Medical Center Boulevard, Winston-Salem, N.C. 27157, Tel No: 333-716-8666; \\ E-mail:vjohn@wakehealth.edu \\ Nicholas Grissom, Wake Forest School of Medicine, Medical Center Boulevard Winston-Salem, N.C. 27103, Tel No: 9197607704 ; \\ E-mail:ngrissom@wakehealth.edu
}

\begin{abstract}
Purpose: We present four patients that experienced vision loss associated with the use and removal of silicone oil as part of treatment for retinal detachment. Though silicone oil has been previously reported to cause vision loss, we believe these cases represent a distinct pathological mechanism.

Methods: Patients underwent evaluation with a complete ophthalmic examination, optical coherence tomography of the macula, magnetic resonance imaging of the orbits, electroretinogram, and other tests as deemed clinically necessary.

Patients: Four patients were diagnosed with rhegmatogenous retinal detachments and underwent vitrectomy with silicone oil placement as part of their treatment. These patients later developed severe central scotomata, and retained poor central visual acuity after refraction and treatment of identifiable causes of decreased acuity.
\end{abstract}

Conclusion: These four patients experienced significant loss of visual acuity associated with the use or removal of silicone oil in the treatment of retinal detachment.

Visual loss has been previously reported with the use of silicone oil, but in prior literature characteristic changes on optical coherence tomography were noted.

Imaging and other diagnostic modalities performed on these patients did not identify the cause for these patients vision loss and we believe these four cases represent a novel pathologic entity.

\section{Introduction}

Silicone oil is a commonly used adjunctive treatment in vitreoretinal surgery [1]. One setting in which the use of silicone oil tamponade has been prominent is the treatment and management of Rhegmatogenous retinal detachment (RRD). The introduction of silicone oil has proven to be beneficial, as few agents match the effectiveness of silicone oil as an ocular tamponade agent. However, studies over the past decade have reported the incidence of vision loss as a complication associated with the use of silicone oil [1-8]. Most reports suggest that a central scotoma is the most common severe complication seen in this setting, suggesting foveal injury or an optic neuropathy [1]. Initially, it was thought that the inciting event for the development of vision loss was the removal of the silicone oil, though patients have also experienced loss of visual acuity prior to removal of the oil. It remains unclear why the retina is affected by silicone oil, and specifically why the fovea in particular is preferentially injured.

Some studies suggest that pre-existing risk factors such as glaucoma and optic nerve abnormalities define the patient population at risk for severe vision loss [4]. Additionally, multiple studies have assessed the ability of Spectral Domain Optical Coherence Tomography (SD-OCT) as a tool to objectively assess the changes associated with silicone oil associated vision loss [7]. We present four patients that experienced severe vision loss following use of silicone oil as part of their treatment for retinal detachment, and discuss how our findings relate to the current body of research.

\section{Patient 1}

A 53-year-oldman presented with a macula-on retinal detachment of the right eye with a giant retinal tear in 2009. The visual acuity on presentation was 20/20-1 in the right eye. He underwent a Pars-Plana Vitrectomy (PPV) with a scleral buckle, external cryopexy and injection of silicone oil. At one month post op, the Best-Corrected Visual Acuity (BCVA) was 20/50. Three months post-operatively he reported that his vision was much worse, and the visual acuity was 20/200, but he had developed a dense posterior sub capsular cataract. Phacoemulsification with IOL at post op month four did not improve his vision as anticipated. Nine months following the initial retinal detachment repair, the silicone oil was removed, and his BCVA remained at 20/200 postoperatively. Further evaluation revealed a 0.7 log unit relative afferent pupillary defect in the right eye and Humphrey threshold perimetry demonstrated a discrete central scotoma in the right eye (Figure 1). Intraocular Pressure (IOP) was within normal limits. SD-OCT showed a normal foveal contour and thickness with no epiretinal membrane or cystoid 
macular edema. At 15 months post-operatively, the patient's BCVA had improved minimally to 20/125 in the right eye with unremarkable macular OCT. Three years later, the acuity and visual field was unchanged, and OCT of the macula remained normal (Figure 2).

\section{Patient 2}

A 60-year-oldman with history of ocular hypertension presented with an RRD of the left eye in 2014. He underwent vitrectomy with scleral buckle placement and silicone oil injection one week later due to failure of a pneumatic procedure. The choice of oil as tamponade, as opposed to gas, in his surgery was due to his desire to fly back to his home in Colorado. His postoperative BCVA was 20/30 in the left eye initially. He later underwent cataract surgery with silicone oil removal at a different facility three months later and noted a sudden loss of vision resulting in central scotoma in the early postoperative period. His BCVA decreased to CF@ 3', and IOP was elevated at $24 \mathrm{~mm} \mathrm{Hg}$. The slit lamp exam was unremarkable. Funduscopic exam revealed mild inferior retinal thinning and questionable temporal pallor of the optic disc. OCT showed a mild epiretinal membrane and central thickness of 295 um (Figure 3). Full-field Electroretinogram (ERG) revealed lower amplitudes in the left eye from all scotopic and photopic stimuli, but a multifocal ERG did not reveal any abnormalities, and the optic disc was graded as normal by two different neuro ophthalmologists. An MRI of the orbits with and without contrast did not demonstrate any evidence of a retrobulbar optic neuropathy. At four months after oil removal, his vision remained CF.

\section{Patient 3}

A 44 year old woman with history of pathologic myopia presented in 2014 with two retinal tears in the left eye with resultant vitreous hemorrhage. She was initially treated with pneumatic cryopexy with success in the left eye. Two months post-operatively, the patient presented with bilateral RRDs, and was treated with scleral buckle placement and cryopexy in both eyes. Four weeks following the surgery, the patient had a PPV with silicone oil injection in the left eye for recurrent detachment. The patient's BCVA improved from 20/100 pre-op to 20/25 in the left eye after four weeks. Three months after the procedure, the silicone oil was removed from the left eye, after which patient noticed severe loss of central vision and color vision loss, with BCVA of CF@3'. Intraocular pressures remained normal throughout. Slit lamp examination was notable for trace nuclear sclerosis with $3+$ posterior subcapsular cataract. Fundus exam revealed a flat macula with lacquer cracks and no subretinal fluid or fibrosis of the retina. OCT was obtained and central foveal thickness was 278, with normal foveal profile and no CME or SRF were noted (Figure 4). An MRI obtained two months after oil removal revealed no retrobulbar abnormality and the optic nerve was normal in appearance. An RNFL OCT was also obtained, which was unremarkable. At this time, BCVA was 20/400, with E Card @ 6'. Eight months post-operatively the patient's BCVA was $20 / 150+1$

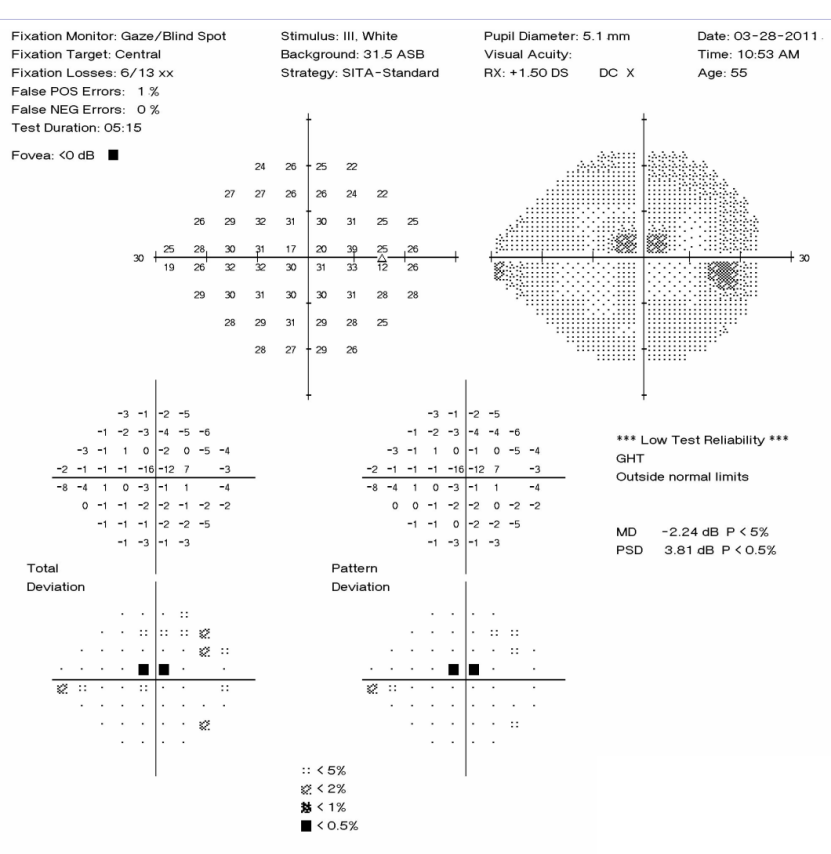

Figure 1: Humphrey threshold 30-2 perimetry shows a discrete central scotoma and decreased foveal threshold.

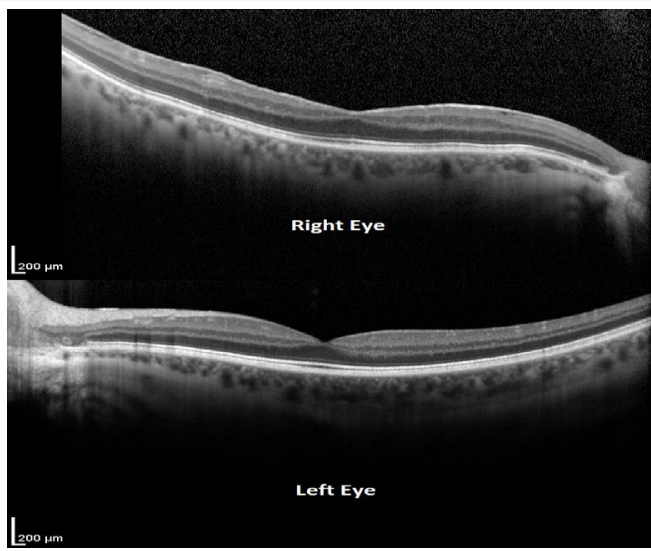

Figure 2: OCT imaging of right eye demonstrates mild epiretinal membrane with normal appearance of the outer retina. We have included his left normal OCT for comparison.

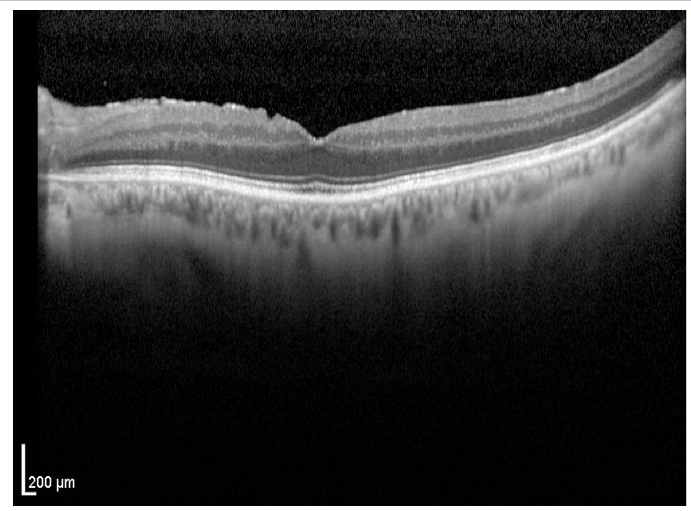

Figure 3: OCT of left macula highlighting normal retinal architecture. 


\section{Patient 4}

A 46 year old female with history of high myopia presented in 2014 with bilateral inferior macula-involving, fovea-threatening retinal detachments that appeared chronic in nature. She had a BCVA of $20 / 25$ on presentation. She underwent cryopexy and scleral buckle placement in the right eye one week after presentation. Following the procedure, persistent sub-retinal fluid was noted in the periphery, so a PPV was performed with placement of silicone oil. Two months postoperatively, BCVA was 20/30 under oil. One year later, the patient presented again with BCVA of CF @ 3' and was found to have a severe posterior subcapsular cataract. Both the cataract and silicone oil were removed at this time. These procedures failed to improve the patient's visual acuity, and at one month post-op the BCVA remained CF @6' in the right eye. Funduscopic exam and fundus photography were remarkable for small area of RPE atrophy on inferior peripheral retina.OCT of macula at one month postop revealed a normal myopic foveal contour (Figure 5). An OCT of RNFL was performed, which was normal (Figure 6). An MRI of the orbits was obtained and did not reveal any retrobulbar abnormality and the optic nerve was normal in appearance. Two months post-operatively, the patient's BCVA remained CF @ 6'.

\section{Discussion}

All four of the patients had acute central visual loss following removal of silicone oil, which was a component of their treatment for retinal detachment. In these patients the diagnostic workup was unable to identify a cause for the decreased vision. In published literature, Errera et al [3] were consistently able to identify abnormalities in patients with visual loss due to silicone oil tamponade on SD-OCT; typically hyper reflective areas adjacent to the retina that they believe represent bubbles of emulsified silicone oil. Shalchi et al [7] also identified microcystic macular changes in the inner nuclear layer of affected eyes on SD-OCT, in addition to noting a focal loss of the papillofoveal projection. This is significant because no such findings were noted in our four patients. Notably, only one of the patients included in this case report had an IOP spike at any point during the workup, and the only evidence of any optic neuropathy was the RAPD seen in Patient 1

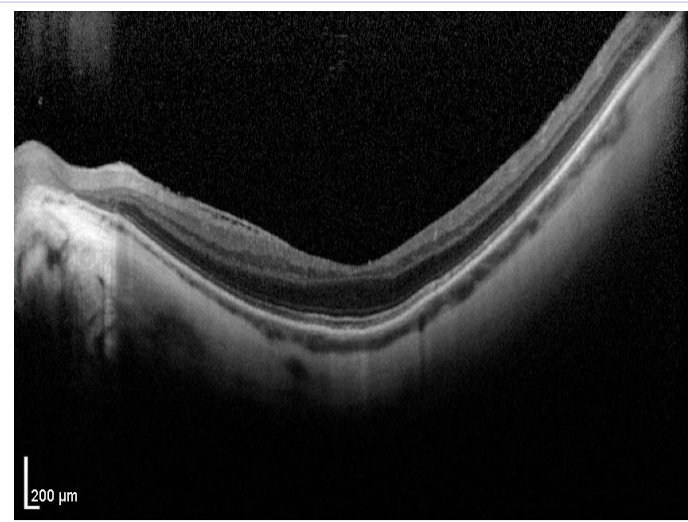

Figure 4: OCT of left eye demonstrating a myopic fundus. The retinal layers are normal and intact.

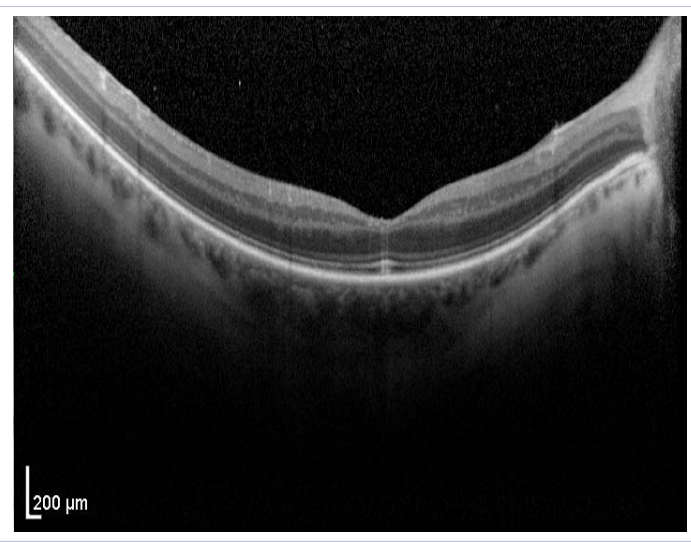

Figure 5: OCT of right macula demonstrating myopic fundus with normal retinal architecture.

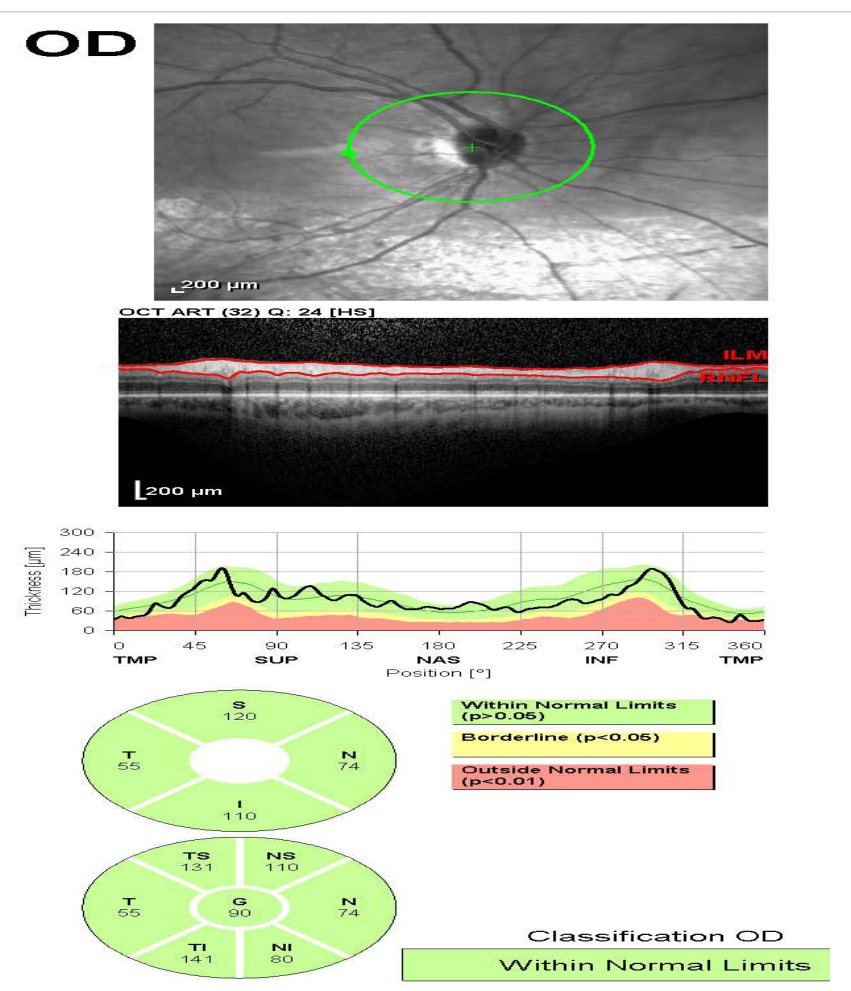

Figure 6: Normal OCT of retinal nerve fiber layer in the right eye.

Several theories have been proposed about the mechanism by which silicone oil results in visual loss. Animal studies were the first to demonstrate that silicone oil penetrated the internal limiting membrane and caused loss of horizontal and bipolar cell processes [1]. A study performed by Williams et al. [8] delineated the modern theories about how silicone oil causes injury. Perhaps the most prominent idea in current literature is the "vitreous potassium sink" theory, which suggests that the silicone oil tamponade disrupts the natural potassium reservoir provided by the vitreous, allowing intraretinal potassium spikes that can injure the outer layers of the fovea. An alternative idea suggests that the silicone oil droplets themselves migrate subretinally, which can result in optic neuropathy that is similar 
in phenotype to that caused by multiple sclerosis and Leber's optic neuropathy [4]. Nicholson et al suggest that accumulation of oil droplets in the prefoveal region is not the likely mechanism of injury, a conclusion that is consistent with many other studies that have not found a clear correlation between the length of time silicone oil tamponade was in place with the injury rate or severity [6]. Most modern data suggests that the end result of the silicone oil tamponade is neuronal cell loss in the macula. This results in a measureable reduction in inner retinal thickness, and is the physiologic defect that results in the visual changes we observed [2]. The cases we present here are significant because previous reports of silicone oil toxicity have demonstrated characteristic OCT findings, while these four patients do not have OCT abnormalities that explain their visual loss. We believe these patients represent a different pathologic entity from those reported in the literature, and with current imaging modalities we do not have an explanation of the etiology.

Though the techniques needed to establish how silicone oil results in visual loss are well-described, currently the pathophysiology of the condition is not well-reported in literature. It is unclear if the microcystic macular changes that have been observed are also present in unaffected eyes but are asymptomatic, or if they are an integral part of the pathogenesis of the visual loss. The rarity of this complication makes studies with significant power difficult to create, so a larger sample size may be necessary to adequately study this phenomenon.

\section{References}

1. Newsom RSB, Johnston R, Sullivan PM, Aylward GB, Holder GE, Gregor ZJ. Sudden visual loss after removal of silicone oil. Retina. 2004;24(6):871-877.

2. Christensen UC, La Cour M. Visual loss after use of intraocular silicone oil associated with thinning of inner retinal layers. Acta Ophthalmol. 2012;90(8):733-737. doi:10.1111/j.1755-3768.2011.02248.x.

3. Errera MH, Liyanage SE, Elgohary M, Day AC, Wickham L, Patel PJ, et al. Using spectral-domain optical coherence tomography imaging to identify the presence of retinal silicone oil emulsification after silicone oil tamponade. Retina. 2013;33(8):1567-1573. doi:10.1097/ IAE.0b013e318287d9ea.

4. Grzybowski A, Pieczynski J, Ascaso FJ. Neuronal complications of intravitreal silicone oil: An updated review. Acta Ophthalmol. 2014;92(3):201-204. doi:10.1111/aos.12212.

5. Herbert EN, Habib M, Steel D, Williamson TH. Central scotoma associated with intraocular silicone oil tamponade develops before oil removal. Graefe's Arch Clin Exp Ophthalmol. 2006;244(2):248-252. doi:10.1007/s00417-005-0076-6.

6. Nicholson BP, Bakri SJ. Silicone Oil Emulsification at the Fovea as a Reversible Cause of Vision Loss. JAMA Ophthalmol. 2015;133(4):484486. doi: 10.1001/jamaophthalmol.2014.5505.

7. Shalchi Z, Mahroo OA, Shunmugam M, Mohamed M, Sullivan PM, Williamson TH. Spectral Domain Optical Coherence Tomography Findings in Long-Term Silicone Oil-Related Visual Loss. Retina. 2015;35(3):555-563. doi:10.1097/IAE.0000000000000325.

8. Williams PD, Fuller CG, Scott IU, Fuller DG, Flynn HW. Vision loss associated with the use and removal of intraocular silicone oil. Clin Ophthalmol. 2008;2(4):955-959. 